\title{
Role of Correct Coding for Interventional Techniques
}

\author{
Laxmaiah Manchikanti, MD
}

There has been enormous emphasis on the description and definition of what the physician does for and to the patient, with fraud and abuse evolving as an important aspect of interventional pain medicine. Compliance with the laws and regulations encompassing documentation with coding, billing, and collections, and medical records, is crucial in today's interventional pain medicine practices. The Health Insurance Portability and Accountability Act of 1996, provided the Office of Inspector General and the Federal Bureau of Investigations with broad powers and directed them to identify and prosecute health-care fraud and abuse.

The National Correct Coding Council was created by Centers for Medicare and Medicaid Services to help ensure that providers across various jurisdictions receive like payments for the same services, use the same codes and provide simi-

Correct coding and billing, appropriate documentation, and the threat of fraud and abuse are part of the daily lives of physicians practicing interventional pain medicine. There has been enormous emphasis on the description and definition of what the physician does for and to the patient. Compliance with the laws and regulations encompassing documentation with coding, billing, and collections, and medical records, is of crucial importance in today's interventional pain medicine practices. The Balanced Budget Act of 1997 empowered and directed the Centers for Medicare and Medicaid Services (CMS), formerly the Health Care Financing Administration (HCFA), to develop a payment system based upon physician work, and guidelines to document that work. The Kennedy-Kassebaum Health Reform Bill of 1996, also known as the Health Insurance Portability and Accountability Act (HIPAA), in addition provided the Office of Inspector General (OIG) and the Federal Bureau of Investigations (FBI) with broad powers and directed them to identify and prosecute health-care fraud and abuse. However, it is the responsibility of CMS (HCFA) to make certain that uniform payment policies and

From Pain Management Center of Paducah, Paducah, Kentucky. Dr. Manchikanti is Medical Director at the Pain Management Center of Paducah. Address correspondence: Laxmaiah Manchikanti, MD, 2831 Lone Oak Road, Paducah, Kentucky 42003. E-mail: drm@asipp.org lar documentation for services performed.

As a direct outgrowth of the National Correct Coding Council's work, the Centers for Medicare and Medicaid Services established the National Correct Coding Policy in 1996 and eventually implemented the Correct Coding Initiative (CCI) to identify and isolate inappropriate coding, unbundling, and other irregularities in coding. Multiple versions of National Correct Coding Policies have been released in the form of National Correct Coding manuals ranging from version 5.0 to 7.2. This review discusses various aspects of correct coding in interventional pain medicine.

Keywords: Fraud, abuse, correct coding, interventional pain medicine, comprehensive codes, component codes, mutually exclusive codes

procedures are followed by all carriers, except in those instances where carriers are authorized to make local medical review policy. The National Correct Coding Council (NCCC) was created by CMS (HCFA) to help ensure that providers across various jurisdictions receive like payments for the same services (altered only by mandated geographic adjustments), use the same codes and provide similar documentation for services performed.

\section{HISTORY}

In December 1989, Omnibus Budget Reconciliation Act, Section 6102 of P.L. 101-239, amended title XVIII of the Social Security Act, dealing with payment for physicians' services. Addition of this new section (section 1848) provided a resource-based relative value scale (RBRVS) fee schedule in 1992, replacing the reasonable-charge mechanism of actual, customary and prevailing charges. As a direct outgrowth of the NCCC's work, CMS (HCFA) established the National Correct Coding Policy in 1996 and eventually implemented the Medicare Correct Coding Initiative (CCI) to identify and isolate inappropriate coding, unbundling, and other irregularities in coding. Multiple versions of National Correct Coding Policies have been released in the form of National Correct Coding manuals ranging from version 5.0 to 7.2. In addition, CMS (HCFA) also has utilized unpublished coding edits referred to as "black-box 
edits" which essentially are a system of payment denials to be used by carriers based on commercial utilization guidelines; carriers refuse to give beneficiaries and providers useful, complete information as to the rationale for these denials on the grounds that the guidelines used are "property" and, thus, confidential. Along with published CCIs, black-box edits were updated frequently and have become one of the most contentious issues related to Medicare and private insurance reimbursement in today's practice of medicine. With a multitude of arguments on the issue, black-box edits were finally eliminated in the year 2000 .

\section{NATIONAL CORRECT CODING POLICY}

The NCCC's policies are based on established coding conventions defined in the American Medical Association's (AMA) Current Procedural Terminology (CPT) manual, national and local policies and edits, coding guidelines developed by national societies, analysis of standard medical and surgical practice, and reviews of current coding practices.

Correct coding essentially means reporting a group of procedures with appropriate comprehensive code. Under the CCI, CMS (HCFA) has developed general policies that define the coding principles and edits that apply to procedure and service codes. Prior to development of CCI edits, Medicare Part B carriers included in their claims-processing systems various computerized edits to detect improper coding of procedures, which at the time was designated as fragmentation.

In consideration of monumental changes in outpatient coding with multiple proposals in 1999, 2000 and 2001 by CMS (HCFA); development of multiple new codes and revision of codes along with deletion of codes by AMA for CPT 2000 and 2001; and use of modifiers and ambulatory payment classifications by CMS (HCFA); CMS officials have acknowledged that they have assigned over 105,000 coding edits to edit approximately 5,600 CPT codes Part B carriers use to analyze claims. These edits will be applied to physician services, services provided in ambulatory surgical centers, and hospital outpatient department claims.

Since the installation of the Correct Coding Edits, which went into effect January 1, 1996, Medicare claims of inappropriate coding practices by providers and, thus, rejections have multiplied, resulting in substantial cost savings for the Medicare program. With the passage of the Health Insurance Portability and Accountability Act of 1996 (HR
3103), correct coding practices became part of the requirement to obtain proper payment from Medicare, rather than an option. The CMS's (HCFA) current coding policies and edits apply when the same provider bills for all the procedures involved, or when the services for the same beneficiary were provided on the same day.

\section{GENERAL CORRECT CODING POLICIES}

In order for the CCI to be effective, it is essential that the coding description accurately describes what actually transpired at each patient encounter. A multitude of codes reflect the wide spectrum of services provided by various providers, and many medical services can be rendered by different methods and combinations of various procedures. Hence, multiple codes describing similar services are frequently necessary to accurately reflect the particular service a physician performs. However, when multiple procedures are performed at the same session, the procedure and postprocedure work do not have to be repeated for each procedure; and, therefore, a comprehensive code describing the multiple services commonly performed together can be used. Many activities which are integral to a procedure are considered as generic activities and are assumed to be included as acceptable medical/surgical practice and, while they could be performed separately, they should not be considered as such when a code narrative is defined. Hence, all services integral to accomplishing a procedure will be considered to be included in that procedure and, therefore, will be considered a component and part of the comprehensive code $(2,3)$.

\section{Standards of Medical/Surgical Practice}

Many of the provider activities during a procedure are integral to a procedure and termed generic activities, which are assumed to be included as acceptable medical/ surgical practice, considered included in that procedure and considered a component of the procedure.

\section{Medical/Surgical Package}

Over the years the CPT manual has grown to accommodate the expanding variety of surgical, diagnostic, and therapeutic surgical, as well as nonsurgical procedures performed. In general, most services include associated preprocedure and postprocedure work; when performed at a single patient encounter, the preprocedure and postprocedure work is relatively fixed, regardless of the number of services actually performed at each session. For interventional pain procedures, some general guidelines can be developed. 


\section{Add-on Codes}

The CPT coding system identifies certain codes that are submitted with other codes. These codes are identified generally with a statement such as, "List separately in addition to code for primary procedure in parentheses," but sometimes the supplemental code is to be used only with certain primary codes that are identified in parentheses. The purpose of these CPT codes is to enable providers to separately identify a service that is performed in certain situations as an additional service (3-5).

\section{Modifiers}

Modifiers are attached to the end of a code to indicate that a service or procedure described in the code definition has been modified by some circumstance. However, explicit understanding of the purpose of each modifier is required prior to its usage. It is also essential to recognize that modifiers may be different for each locality. In addition, it is essential to understand the specific meaning of the modifier for the payer to which a claim is being submitted before using it.

Within the context of multiple-services reporting, without the addition of an appropriate modifier, it will appear that providers are engaging in the practice of "unbundling." The appropriate use of modifiers indicates that the services were performed under circumstances which did not involve this practice at all.

\section{Excluded Services}

Even though the Medicare program has identified some services as "excluded services," they have not been included in CCI audits.

\section{CPT Procedure Code Definition}

All procedures described by the CPT code narrative for the comprehensive code should have been performed, and only the single CPT code most accurately describing the procedure should be reported (1-3).

\section{CPT Coding Manual Instruction Guidelines}

Each section of the CPT coding manual includes instructions that are unique to that section, in addition to the general instructions provided. These directions are not all inclusive of, nor limited to, definitions of terms; modifiers; unlisted procedures or services; special or written re- ports; and details about reporting separate, multiple or starred procedures and qualifying circumstances $(2,4,5)$. These instructions define items or provide explanations that are necessary to appropriately interpret and report the procedures or services and to define terms that apply to a particular section.

\section{Separate Procedures}

The "separate procedure" notation in the CPT manual identifies a procedure or service that can be performed independently but that, when performed as an integral part of the comprehensive procedure, should not be reported separately.

\section{Designation of Sex}

Many procedure codes have a sex designation within their narrative; however, these codes do not apply to the practice of interventional pain medicine.

\section{Family of Codes}

The CPT manual describes certain codes that include two or more component codes that should not be reported separately, as these are considered members of a code family and included in a more comprehensive code. As such, comprehensive codes include certain services that are separately identified by other component codes. Although component codes as members of the comprehensive code family represent parts of the procedure that should not be listed separately when the complete procedure is performed, the component codes are considered individually if the procedures they describe are performed independently of the complete procedure. If this is not the case, all services listed in the comprehensive codes will be considered to make up the total service.

\section{Most Extensive Procedures}

The CMS (HCFA) established policy is that, for a code which applies to a procedure that can be performed at different levels of complexity, only the most extensive service, encompassing the comprehensive code actually performed, should be reported. Thus, when procedures are performed together that are basically the same, or performed on the same side but qualified by an increased level of complexity, the less extensive procedure is included in the more extensive procedure. Hence, the procedure viewed as the most complex should be reported. 


\section{Sequential Procedures}

Sequential procedures or a successful procedure is identified in this policy, which identifies codes for procedures that are often performed in sequence or for procedures involving an initial approach followed by a more invasive procedure during the same encounter. Only the procedure that successfully accomplishes the expected result is reported, with a less extensive procedure bundled into the more extensive one.

\section{With or Without Procedures}

Certain codes in the CPT manual identify a procedure that can be performed with or without certain services. It is contradictory to report code combinations in which one code represents a procedure that includes a certain service and the other code represents the procedure without that service.

\section{Laboratory Panels}

Medical necessity for laboratory evaluation must be established. In addition, an interventional pain specialist should pay close attention to comprehensive panel codes that include multiple component tests.

\section{Unlisted Services or Procedures}

Multiple sections in the CPT manual list certain codes that end in "99" or "9," in a few cases used to report a service that is not described in any code listed elsewhere in the CPT manual. This facilitates advances in technology, or physician expertise with new procedures when a CPT code may not have been assigned to a procedure when it is first introduced as accepted treatment.

\section{Mutually Exclusive Codes}

Mutually exclusive codes are codes for procedures that cannot reasonably be performed in the same session.

\section{Correct Coding and its Modifier Indicator}

An appropriate modifier must be used with a code for which a modifier is appropriate. This code may be a column 1 or column 2 code. The definition of a modifier, according to the CPT manual, is as follows: "a modifier provides the means by which the reporting physician or provider can indicate that a service or procedure that has been performed has been altered by some specific circumstance but not changed in its definition or code."

Thus, the correct coding file formats have been presumably expanded to include a modified indicator for both the mutually exclusive code and the comprehensive component code tables. The Correct Coding Edits modifier indicators are " 0, , " 1 ," or "9."

- A " 0 " indicates that there are no circumstances in which a modifier would be appropriate. The services represented by the code combination will not be paid separately.

- A " 1 " indicates that a modifier is allowed in order to differentiate between the services provided.

- A "9" indicator is used for all code pays having a deletion date the same as their effective date.

\section{INCORRECT CODING}

Incorrect coding is defined as the intentional or unintentional billing of multiple procedure codes for a group of procedures that are covered by a single comprehensive code. Incorrect coding includes both unbundling and upcoding. Various types of incorrect coding examples include:

- Fragmenting one service into component parts and coding each component as if it were a separate service;

- Reporting separate codes for related services when the comprehensive code includes all related services;

- Breaking out bilateral procedures when one code is appropriate;

- Downcoding a service in order to use an additional code when one higher-level, more comprehensive code is appropriate; and

- Separating a surgical approach from a major surgical service.

Consequences of inaccurate coding are disastrous, as shown in Table 1.

\section{CORRECT CODING POLICIES FOR INTERVENTIONAL TECHNIQUES}

Various commonly utilized interventional techniques in pain management are described here with comprehensive, component, and mutually exclusive codes. However, if there is a question about coding, its comprehensive nature, or mutual exclusion, physicians and other providers are urged to contact local Medicare carriers or third-party 
Table 1. Consequences of inaccurate coding and billing in the order of importance

\begin{tabular}{ll}
\hline Denied claims & Prison \\
Trigger for a review & Exclusion \\
Returned claims & Sanctions \\
Suspended claims & Fines \\
Downcoding & False Claims \\
\hline
\end{tabular}

payers. The CCI 7.2 involves interventional pain medicine, with, overall, approximately 5000 new CCI CodePair edits that went into effect on July 1, 2001(2,4,5). Among the hardest hit by these changes will be interventional pain physicians, even though almost all of the specialties are affected. Tables 2 to 4 show important aspects of correct coding; most commonly used comprehensive, component and mutually exclusive codes; and the crosswalk to comprehensive and component codes.

\section{COMPREHENSIVE, COMPONENT ANDMUTUALL EXCLUSIVE CODES}

- CPT - 20550 injection, tendon sheath, ligament, trigger points or ganglion cyst; a comprehensive code which also includes several component codes as follows:

- 10160 puncture aspiration of abscess, hematoma or cyst
- 11900 injection, intralesional; up to and including seven lesions

- 11901 injection, intralesional; more than seven lesions

- 12032 layer closure of wounds of scalp, axillae, trunk, and/or extremities; $2.6 \mathrm{~cm}$ to $7.5 \mathrm{~cm}$

- 12042 layer closure of wounds of neck, hands, feet, and/or external genitalia; $2.6 \mathrm{~cm}$ to $7.5 \mathrm{~cm}$

- 20500 injection of sinus tract; therapeutic (separate procedure)

- 29075 application of halo type body cast; elbow to finger (short arm)

- 29105 application of long arm splint (shoulder to hand)

- 29125 application of short arm splint (forearm to hand); static

- 29130 application of finger splint; static

- 29220 strapping; low back

- 29260 strapping; elbow or wrist

- 29405 application of short leg cast (below knee to toes)

- 29425 application of short leg cast (below knee to toes); walking or ambulatory type

- 29450 application of clubfoot cast with molding or manipulation, long or short leg

- 29515 application of short leg splint (calf to foot)

- 29530 strapping; knee

- 29540 strapping; ankle

- 29550 strapping; toes

Table 2. Ten important aspects of correct coding for interventional techniques

1. Understand comprehensive and mutually exclusive codes

2. Do not use component and mutually exclusive codes

3. Watch for exclusion of fluoroscopy codes:

$76000 \quad 76003$

$76001 \quad 76005$

4. Do not bill for combined epidural procedures in the same region, eg, interlaminar and transforaminal

5. Do not bill epidurals with discograms. Discograms are noncovered procedures for ASC facility fees

6. Do not bill for nerve blocks and neurolytic blocks in the same region

7. Do not bill trigger point injections with somatic nerve blocks (CPT 64400-64445)

8. Do not bill trigger point injections with facet joint nerve blocks or transforaminal epidural injections

9. Do not bill medium and large joint injections with:

Sacroiliac joint injection

All types of epidurals

Facet joint injections

10. Do not bill small joint injections with:

Sacroiliac joint injection

Facet joint injections 
Table 3. Illustration of most commonly used interventional techniques showing component and mutually exclusive codes

\begin{tabular}{|c|c|c|}
\hline Comprehensive code & Component codes & $\begin{array}{l}\text { Mutually } \\
\text { exclusive codes }\end{array}$ \\
\hline 27096 - sacroiliac joint injection & 20600 , to $20610,69990,76000$ to 7600390782 & NA \\
\hline 62263 - adhesiolysis & $\begin{array}{l}62281,62282,62284,62310,62311,62318,62319, \\
64479,64483,64722,69990,76000,76003,76005\end{array}$ & NA \\
\hline 62290 - lumbar discography & $62311,62319,64483,69990,76005$ & NA \\
\hline 62291 - cervical discography & $62310,62318,64479,69990,76005$ & NA \\
\hline 62310 - cervical epidural & $20605,20610,36140,62284,69990,76000$ to 76003 & 64479 \\
\hline 62311 - lumbar epidural & $20605,20610,36140,62284,69990,76000$ to 76003 & 64483 \\
\hline $\begin{array}{l}64470 \text { - cervical/thoracic facet } \\
\text { joint nerve blocks }\end{array}$ & $20550,20600,20605,20610,36140,76000$ to 76003 & NA \\
\hline $\begin{array}{l}64475 \text { - lumbar facet joint nerve } \\
\text { blocks }\end{array}$ & $\begin{array}{l}20550,20600,20605,20610,36140,69990,76000 \\
\text { to } 76003\end{array}$ & NA \\
\hline $\begin{array}{l}64479 \text { - cervical/thoracic } \\
\text { transforaminal epidural }\end{array}$ & $20550,20605,20610,36140,69990,76000$ to 76003 & NA \\
\hline $\begin{array}{l}64483 \text { - lumbar transforaminal } \\
\text { epidural }\end{array}$ & $20550,20605,20610,36140,76000$ to 76003 & NA \\
\hline 64620 - intercostal neurolysis & $64420,64421,69990$ & NA \\
\hline 64622 - lumbar facet neurolysis & $64475,69990,76000$ to 76003 & NA \\
\hline 64626 - cervical facet neurolysis & $64470,69990,76000$ to 76003 & NA \\
\hline 64640 - peripheral nerve neurolysis & $\begin{array}{l}64405,64408,64410,64415,64417,64418,64425, \\
64435,64445,64450,69990\end{array}$ & NA \\
\hline
\end{tabular}

$\mathrm{NA}=$ not applicable

- 29580 strapping; Unna boot

- 29590 Denis-Browne splint strapping

- 64550 application of surface (transcutaneous) neurostimulator

- 64714 neuroplasty, major peripheral nerve, arm or leg; lumbar plexus

- 69990 operating microscope

- 72240 myelography, cervical radiological supervision and interpretation

- 72265 myelography, lumbosacral, radiological supervision and interpretation

- 72295 diskography, lumbar, radiological supervision and interpretation

- 76000-76003 fluoroscopy codes

- 87102 culture, fungi (mold or yeast) isolation, with presumptive identification of isolates; other source (except blood)

- 90780 intravenous (IV) infusion for therapy

- 90781 IV infusion for therapy

- 90782 subcutaneous or intramuscular (IM) injection
- 95900 nerve conduction, amplitude and latency/ velocity study, each nerve; motor, without F-wave study

Mutually exclusive code:

- 11010 debridement and removal of foreign material associated with open fractures; skin and subcutaneous tissues.

- CPT 20600 - arthrocentesis, aspiration and/or injection; small joint, bursa or ganglion cyst, eg, fingers, toes; a comprehensive code which also includes several component codes as follows:

- 10060 incision and drainage of abscess

- 10061 incision and drainage of abscess; complicated

- 10140 incision and drainage of hematoma or seroma

- 10160 puncture aspiration of abscess or hematoma

- 11719 trimming of nondystrophic nails, any number 
Table 4. Illustration of most commonly used interventional techniques showing component and mutually exclusive codes

\begin{tabular}{|c|c|c|c|c|c|c|c|c|c|c|c|}
\hline \multirow{2}{*}{$\begin{array}{l}\text { Component } \\
\text { Codes }\end{array}$} & \multicolumn{11}{|c|}{ Comprehensive Code } \\
\hline & 27096 & 62263 & 62281 & 62282 & 62287 & 62290 & 62291 & 62310 & 62311 & 62318 & 62319 \\
\hline 20600 & $X$ & & & & & & & & & & \\
\hline 20605 & $x$ & & & & & & & $x$ & $x$ & $x$ & $x$ \\
\hline 20610 & $X$ & & & & & & & $X$ & $x$ & $x$ & $X$ \\
\hline 36140 & & & & & & & & $X$ & $x$ & $x$ & $x$ \\
\hline 62270 & & & $X$ & $X$ & & & & & & $x$ & $X$ \\
\hline 62273 & & & $X$ & $X$ & & & & & & $\mathrm{Y}$ & $\mathrm{Y}$ \\
\hline 62284 & & $X$ & $X$ & & & & & $X$ & $x$ & $X$ & $x$ \\
\hline 62310 & & $x$ & $x$ & & $x$ & & $x$ & & & $x$ & \\
\hline 62311 & & $X$ & & $x$ & $x$ & $x$ & & & & & $x$ \\
\hline 62318 & & $x$ & $x$ & & $x$ & & $x$ & & & & \\
\hline 62319 & & $X$ & & $X$ & $x$ & $X$ & & & & & \\
\hline 64479 & & $x$ & $X$ & & $x$ & & $x$ & $\mathrm{Y}$ & & $\mathrm{Y}$ & \\
\hline 64483 & & $X$ & & $x$ & $x$ & $x$ & & & $\mathrm{Y}$ & & $\mathrm{Y}$ \\
\hline 69990 & $X$ & $X$ & $x$ & $x$ & $x$ & $\mathrm{X}$ & $x$ & $x$ & $x$ & $x$ & $x$ \\
\hline 76000 & $X$ & $X$ & $X$ & $X$ & $x$ & & & $X$ & $X$ & $x$ & $X$ \\
\hline 76001 & $X$ & & $X$ & $X$ & $x$ & & & $X$ & $x$ & $x$ & $x$ \\
\hline 76003 & $X$ & $X$ & $X$ & $X$ & & & & $X$ & $X$ & $X$ & $X$ \\
\hline 76005 & & $x$ & & & $X$ & $X$ & $x$ & & & & \\
\hline
\end{tabular}

\section{$X$ - Component Codes}

\section{Y - Mutually Exclusive Codes}

20600-small joint injection

20605-intermediate joint injection

20610-major joint injection

27096-sacroiliac joint injection

36140-introduction of needle or intracatheter, extremity artery;

62263-percutaneous adhesiolysis

62270-spinal puncture, lumbar, diagnostic

62273-injection, epidural, of blood or clot patch

62281-neurolytic epidural, cervical or thoracic

62282-neurolytic epidural, lumbar, sacral (caudal)

62284-injection procedure for myelography

62287-disc decompression procedure

62290-lumbar discography 62291-cervical discography

62310-cervical epidural

62311-lumbar epidural

62318-catheter placement, continuous infusion or intermittent bolus; epidural or subarachnoid; cervical or

thoracic

62319-catheter placement, continuous infusion or intermittent bolus; epidural, lumbar, sacral (caudal)

64479 - cervical/thoracic transforaminal epidural

64483 - lumbar transforaminal epidural

69990 - use of operating microscope

76000 - fluoroscopy

76001 - fluoroscopy

76003 - fluoroscopy

76005 - fluoroscopy 
- 20500 injection of sinus tract; therapeutic (separate procedure)

- 20550 injection, tendon sheath, ligament or trigger points

- 29065 application of halo-type body cast; long arm

- 29075 application of halo-type body cast; short arm

- 29085 application of halo-type body cast; hand and lower forearm

- 29105 application of long arm splint

- 29125 application of short arm splint; static

- 29130 application of finger splint; static

- 29260 strapping; elbow or wrist

- 29280 strapping; hand or finger

- 29365 application of cylinder cast

- 29405 application of short leg cast

- 29425 application of short leg cast; walking or ambulatory type

- 29505 application of long leg splint

- 29515 application of short leg splint

- 29540 strapping; ankle

- 29550 strapping; toes

- 29580 strapping; Unna boot

- 29590 Denis-Browne splint strapping

- 64450 injection, anesthetic agent; other peripheral nerve or branch

- 64704 neuroplasty; nerve of hand or foot

- 64708 neuroplasty; major peripheral nerve, arm or leg; other than specified

- 69990 use of operating microscope

- 72240 myelography, cervical radiological supervision and interpretation

- 72265 myelography, lumbosacral, radiological supervision and interpretation

- 76000-76003 fluoroscopy codes

- 90780 IV infusion for therapy/diagnosis

- 90782 subcutaneous or IM injection

- 95900 nerve conduction, each nerve; motor, without F-wave study

- G0127 trimming of dystrophic nails, any number

Mutually exclusive code:

- 11010 debridement and removal of foreign material associated with open fractures; skin and subcutaneous tissues

- CPT 20605 - arthrocentesis, aspiration and/or injection; intermediate joint, bursa or ganglion cyst, eg, temporomandibular, acromioclavicular, wrist, elbow or ankle, olecranon bursa; a comprehen- sive code which also includes several component codes as follows:

- 10060 incision and drainage of abscess

- 10061 incision and drainage of abscess; complicated

- 10140 incision and drainage of hematoma or seroma

- 10160 puncture aspiration of abscess or hematoma

- 11900 injection, intralesional; up to and including seven lesions

- 12011 simple repair of superficial wounds of face, ears, eyelids, nose, lips and/or mucous membranes; $2.5 \mathrm{~cm}$ or less

- 15852 dressing change (for other than burns) under anesthesia (other than local)

- 20550 injection, tendon sheath, ligament or trigger points

- 29065 application of halo-type body cast; long arm

- 29075 application of halo-type body cast; short arm

- 29085 application of halo-type body cast; hand and lower forearm

- 29105 application of long arm splint

- 29125 application of short arm splint; static

- 29126 application of short arm splint; dynamic

- 29240 strapping; shoulder, eg, Velpeau

- 29260 strapping; elbow or wrist, eg, Velpeau

- 29405 application of short leg cast

- 29425 application of short leg cast; walking or ambulatory type

- 29445 application of rigid total-contact leg cast

- 29505 application of long leg splint

- 29515 application of short leg splint

- 29540 strapping; ankle

- 29580 strapping; Unna boot

- 29590 Denis-Browne splint strapping

- 29705 removal of bivalving; full arm or full leg cast

- 64450 injection, anesthetic agent; other peripheral nerve or branch

- 64550 application of surface (transcutaneous) neurostimulator

- 64704 neuroplasty; nerve of hand or foot

- 69990 use of operating micros

- 76000- 76003 fluoroscopy codes

- 90780 IV infusion for therapy/diagnosis

- 90782 subcutaneous or IM injection

- 95900 nerve conduction, each nerve; motor, without F-wave study 
Mutually exclusive code:

- 11010 debridement and removal of foreign material associated with open fractures; skin and subcutaneous tissues

- CPT 20610 - arthrocentesis, aspiration and/or injection; major joint or bursa, eg, shoulder, hip, knee joint, subacromial bursa; a comprehensive code which also includes several component codes as follows:

- 10060 incision and drainage of abscess

- 10061 incision and drainage of abscess; complicated

- 10140 incision and drainage of hematoma or seroma

- 10160 puncture aspiration of abscess or hematoma

- 11900 injection, intralesional; up to and including seven lesions

- 12001 simple repair of superficial wounds; 2.5 $\mathrm{cm}$ or less

- 12002 simple repair of superficial wounds; 2.6 $\mathrm{cm}$ to $7.5 \mathrm{~cm}$

- 12020 treatment of superficial wound dehiscence; simple closure

- 12031 layer closure of wounds; $2.5 \mathrm{~cm}$ or less

- 12044 layer closure of wounds; $7.6 \mathrm{~cm}$ to 12.5 $\mathrm{cm}$

- 15851 removal of sutures under anesthesia

- 20500 injection of sinus tract; therapeutic

- 20501 injection of sinus tract; diagnostic

- 20550 injection, tendon sheath, ligament, trigger points or ganglion cyst

- 29065 application of long arm cast

- 29075 application of short arm cast

- 29085 application of cast; hand and lower forearm

- 29105 application of long arm splint

- 29125 application of short arm splint

- 29130 application of finger splint

- 29240 strapping; shoulder

- 29260 strapping; elbow or wrist

- 29345 application of long leg cast

- 29355 application of walking long leg cast

- 29365 application of cylinder cast

- 29405 application of short leg cast

- 29425 application of walking short leg cast

- 29505 application of long leg splint

- 29515 application of short leg splint

- 29530 strapping; knee
- 29540 strapping; ankle

- 29580 strapping; Unna boot

- 64450 injection, anesthetic agent; other peripheral nerve or branch

- 64550 application of surface (transcutaneous) neurostimulator

- 64553 percutaneous implantation of neuro-stimulator electrodes; cranial nerve

- 64718 neuroplasty and/or transposition; ulnar nerve at elbow

- 69990 use of operating micros

- 72255 myelography, thoracic, radiological supervision and interpretation

- 72265 myelography, lumbosacral, radiological supervision and interpretation

- 72295 diskography, lumbar, radiological supervision and interpretation

- 76000-76003 fluoroscopy codes

- 76080 radiologic examination, abscess, fistula or sinus tract study, radiological supervision and interpretation

- 90780 IV infusion for therapy/diagnosis; up to 1 hour

- 90781 IV infusion for therapy/diagnosis; each additional hour, up to 8 hours

- 90782 subcutaneous or IM injection

- 95900 nerve conduction, each nerve; motor, without F-wave study

- G0168 wound closure utilizing tissue adhesive(s) only

Mutually exclusive code:

- 11010 debridement and removal of foreign material associated with open fractures; skin and subcutaneous tissues

- CPT 22520 - percutaneous vertebroplasty; a comprehensive code which also includes several component codes as follows:

- 36005 injection for contrast venography

- 72128 and 72129 computerized tomography (CT) scan with or without contrast

- $\quad 72130$ CT scan without contrast followed with contrast and further sections

- 75872 venography, epidural, and

- 76000 to 76005 fluoroscopy codes

Mutually exclusive codes: None

- CPT 27096 - injection procedure for sacroiliac joint, arthrography; a comprehensive code includes several component codes as follows: 
- 20600 injection; small joint, bursa or ganglion

- 20605 injection, intermediate joint, bursa or ganglion

- 20610 injection, major joint or bursa

- 69990 operating microscope

- 76000-76003 fluoroscopy codes, and

- 90782 administration of therapeutic substance

- CPT 76005 definition includes sacroiliac joint injection described below:

fluoroscopic guidance and localization of needle or catheter tip for spine or paraspinous diagnostic or therapeutic injection procedures (epidural, transforaminal epidural, subarachnoid, paravertebral facet joint nerve or sacroiliac joint), including neurolytic agent destruction

\section{Mutually exclusive codes: None}

- CPT 62263 - percutaneous lysis of epidural adhesions; a comprehensive code, includes the following component codes:

- 62281 injection/infusion of neurolytic substance; (epidural, cervical or thoracic)

- 62282 injection/infusion of neurolytic substance; epidural lumbar, sacral (caudal).

- 62284 injection procedure for myelography

- 62310 injection, single, epidural or subarachnoid; cervical or thoracic

- 62311 injection, single; epidural, lumbar sacral (caudal)

- 62318 catheter placement, continuous infusion or intermittent bolus; epidural, lumbar, sacral (caudal)

- 62319 injection, including catheter placement, continuous infusion or intermittent bolus; epidural, lumbar, sacral (caudal)

- 64479 transforaminal epidural; cervical or thoracic, single level

- 64483 transforaminal epidural; lumbar or sacral, single level

- 64722 decompression; unspecified nerve(s)

- 69990 use of operating microscope

- 76000, 76003, 76005 fluoroscopy codes

Mutually exclusive codes: None

- CPT 62270 - spinal puncture, lumbar, diagnostic; a comprehensive code, includes the following component codes:
- 62273 injection, epidural, of blood or clot patch

- 62311 lumbar, sacral (caudal)

- 64483 transforaminal epidural; lumbar or sacral, single level

- 69990 use of operating microscope

- 76000-76003 fluoroscopy codes

Mutually exclusive codes: None

- CPT 62272 - spinal puncture, therapeutic, for drainage of spinal fluid (by needle or catheter); a comprehensive code, includes the following component codes:

- 62270 spinal puncture, lumbar, diagnostic

- 62273 injection, epidural, of blood or clot patch

- 62310 injection, single, epidural or subarachnoid; cervical or thoracic

- 62311 injection, single; epidural, lumbar sacral (caudal)

- 64479 transforaminal epidural; cervical or thoracic, single level

- 64483 transforaminal epidural; lumbar or sacral, single level

- 69990 use of operating microscope

- 76000-76003 fluoroscopy codes

Mutually exclusive codes: None

- CPT 62273 - injection, epidural, of blood or clot patch; a comprehensive code, includes the following component codes:

- 36000 introduction of needle or intracatheter, vein

- 36140 introduction of needle or intracatheter, extremity artery

- 36410 venipuncture, child over age 3 years or adult, necessitating physician's skill

- 62310 injection, single, epidural or subarachnoid; cervical or thoracic

- 62311 injection, single; epidural, lumbar sacral (caudal)

- 64479 transforaminal epidural; cervical or thoracic, single level

- 64483 transforaminal epidural; lumbar or sacral, single level

- 69990 use of operating microscope

- 76000-76003 fluoroscopy codes

- G0001 routine venipuncture for collection of specimen(s)

Mutually exclusive codes: None 
- CPT 62280 - injection of neurolytic substance with or without other therapeutic substance (subarachnoid); a comprehensive code, includes the following component codes:

- 62270 spinal puncture, lumbar, diagnostic

- 62272 spinal puncture, therapeutic, for drainage of spinal fluid

- 62273 injection, epidural, of blood or clot patch

- 62284 injection procedure for myelography

- 62310 injection, single, epidural or subarachnoid; cervical or thoracic

- 62311 injection, single; epidural, lumbar sacral (caudal)

- 62318 catheter placement, continuous infusion or intermittent bolus; epidural or subarachnoid; cervical or thoracic

- 62319 catheter placement, continuous infusion or intermittent bolus; epidural, lumbar, sacral (caudal)

- 64479 transforaminal epidural; cervical or thoracic, single level

- 64483 transforaminal epidural; lumbar or sacral, single level

- 69990 use of operating microscope

- 76000-76003 fluoroscopy codes

Mutually exclusive codes: None

- CPT 62281 - injection of neurolytic substance with or without other therapeutic substance (epidural, cervical or thoracic); a comprehensive code, includes the following component codes:

- 62270 spinal puncture, lumbar, diagnostic

- 62272 spinal puncture, therapeutic, for drainage of spinal fluid

- 62273 injection, epidural, of blood or clot patch

- 62284 injection procedure for myelography

- 62310 injection, single, epidural or subarachnoid; cervical or thoracic

- 62318 catheter placement, continuous infusion or intermittent bolus; epidural or subarachnoid; cervical or thoracic

- 64479 transforaminal epidural; cervical or thoracic, single level

- 69990 use of operating microscope

- 76000-76003 fluoroscopy codes

Mutually exclusive codes: None

- CPT 62282 - injection of neurolytic substance with or without other therapeutic substance (epidural, lumbar, sacral (caudal)); a comprehensive code, includes the following component codes:

- 62270 spinal puncture, lumbar, diagnostic

- 62272 spinal puncture, therapeutic, for drainage of spinal fluid

- 62273 injection, epidural, of blood or clot patch

- 62311 injection, single; epidural, lumbar sacral (caudal)

- 62319 catheter placement, continuous infusion or intermittent bolus; epidural, lumbar, sacral (caudal)

- 64483 transforaminal epidural; lumbar or sacral, single level

- 69990 use of operating microscope

- 76000-76003 fluoroscopy codes

Mutually exclusive codes: None

- CPT 62284 - injection procedure for myelography and/or computerized axial tomography, spinal (other than C1-2 and posterior fossa); a comprehensive code, includes the following component codes:

- 62270 spinal puncture, lumbar, diagnostic

- 62272 spinal puncture, therapeutic, for drainage of spinal fluid

- 62273 injection, epidural, of blood or clot patch

- 62282 injection/infusion of neurolytic substance; epidural lumbar, sacral (caudal).

- 64479 transforaminal epidural; cervical or thoracic, single level

- 64483 transforaminal epidural; lumbar or sacral, single level

- 69990 use of operating microscope

- 76000-76005 fluoroscopy codes

Mutually exclusive codes: None

- CPT 62287 - aspiration or decompression procedure, percutaneous, of nucleus pulposus of intervertebral disk, any method, single or multiple levels, lumbar, eg, manual or automated percutaneous diskectomy, percutaneous laser diskectomy; a comprehensive code, includes the following component codes:

- 62290 injection procedure for diskography, each level; lumbar

- 62310 injection, single, epidural or subarachnoid; cervical or thoracic

- 62311 injection, single; epidural, lumbar sacral (caudal) 
- 62318 catheter placement, continuous infusion or intermittent bolus; epidural or subarachnoid; cervical or thoracic

- 62319 catheter placement, continuous infusion or intermittent bolus; epidural, lumbar, sacral (caudal)

- 64479 transforaminal epidural; cervical or thoracic, single level

- 64483 transforaminal epidural; lumbar or sacral, single level

- 69990 use of operating microscope

- 76000-76005 fluoroscopy codes

Mutually exclusive codes are as follows:

- 22224 osteotomy of spine, including diskectomy, anterior approach, single vertebral segment (lumbar)

- 22558 arthrodesis, anterior interbody technique, including minimal diskectomy to prepare interspace (other than for decompression, (lumbar))

- 63005 laminectomy with exploration and/or decompression of spinal cord and/or cauda equina, without facetectomy, foraminotomy or diskectomy, one or two vertebral segments (lumbar, except for spondylolisthesis)

- 63017 laminectomy with exploration and/or decompression of spinal cord and/or cauda equina, without facetectomy, foraminotomy or diskectomy, more than 2 vertebral segments (lumbar)

- 63030 laminotomy (hemilaminectomy), with decompression of nerve root(s), including partial facetectomy, foraminotomy and/or excision of herniated intervertebral disk; one interspace (lumbar)

- 63042 laminotomy (hemilaminectomy), with decompression of nerve root(s), including partial facetectomy, foraminotomy and/or excision of herniated intervertebral disk, re-exploration, single interspace (lumbar)

- 63056 transpedicular approach with decompression of spinal cord, equina and/or nerve root(s), single segment (lumbar)

- CPT 62290 - injection procedure for diskography, each level (lumbar); a comprehensive code, includes the following component codes:

- 62311 injection, single; epidural, lumbar sacral (caudal)

- 62319 catheter placement, continuous infusion or intermittent bolus; epidural, lumbar, sacral (caudal)
- 64483 transforaminal epidural; lumbar or sacral, single level

- 69990 use of operating microscope

- 76005 fluoroscopy codes

Mutually exclusive codes: None

- CPT 62291 - injection procedure for diskography, each level (cervical or thoracic); a comprehensive code, includes the following component codes:

- 62310 injection, single, epidural or subarachnoid; cervical or thoracic

- 62318 catheter placement, continuous infusion or intermittent bolus; epidural or subarachnoid; cervical or thoracic

- 64479 transforaminal epidural; cervical or thoracic, single level

- 69990 use of operating microscope

- 76005 fluoroscopy codes

Mutually exclusive codes: None

- CPT 62310 - cervical or thoracic epidural injection; a comprehensive code, includes the following component codes:

- 20605 injection, intermediate joint, bursa or ganglion

- 20610 injection, major joint or bursa

- 36140 introduction of needle or intracatheter, extremity artery

- 62284 injection procedure for myelography

- 69990 use of operating microscope

- 76000-76003 fluoroscopy codes

Mutually exclusive code:

- 64479 cervical or thoracic transforaminal epidural, single level

- CPT 62311 - lumbar (caudal epidural injection); a comprehensive code, includes the following component codes:

- 20605 injection, intermediate joint, bursa or ganglion

- 20610 injection, major joint or bursa

- 36140 introduction of needle or intracatheter, extremity artery

- 62284 injection procedure for myelography

- 69990 use of operating microscope

- 76000-76003 fluoroscopy codes

Mutually exclusive code:

- 64483 lumbar or sacral, transforaminal epidural, single level 
- CPT 62318 - continuous infusion with catheter placement (cervical or thoracic) a comprehensive code, includes the following component code:

- 01996 daily management of epidural or subarachnoid drug administration

- 20605 injection, intermediate joint, bursa or ganglion

- 20610 injection, major joint or bursa

- 36000 introduction of needle or intracatheter, vein

- 36140 introduction of needle or intracatheter, extremity artery

- 36410 venipuncture, child over age 3 years or adult, necessitating physician's skill

- 62270 spinal puncture, lumbar, diagnostic

- 62272 spinal puncture, therapeutic, for drainage of spinal fluid

- 62284 injection procedure for myelography

- 62310 injection, single, epidural or subarachnoid; cervical or thoracic

- 69990 use of operating microscope

- 76000-76003 fluoroscopy codes

Mutually exclusive codes:

- 62273 epidural blood patch

- 64479 cervical transforaminal epidural, single level

- CPT 62319 - injection, catheter placement, lumbar or caudal epidural space; a comprehensive code, includes the following component codes:

- 01996 daily management of epidural or subarachnoid drug administration

- 20605 injection, intermediate joint, bursa or ganglion

- 20610 injection, major joint or bursa

- 36000 introduction of needle or intracatheter, vein

- 36140 introduction of needle or intracatheter, extremity artery

- 36410 venipuncture, child over age 3 years or adult, necessitating physician's skill

- 62270 spinal puncture, lumbar, diagnostic

- 62272 spinal puncture, therapeutic, for drainage of spinal fluid

- 62284 injection procedure for myelography

- 62311 injection, single; epidural, lumbar sacral (caudal)

- 69990 use of operating microscope

- 76000-76003 fluoroscopy codes
Mutually exclusive codes:

- 62273 epidural blood patch

- 64479 cervical transforaminal epidural, single level

- CPT 62350 - implantation, revision or repositioning of tunneled intrathecal or epidural catheter, for long-term medication administration via an external pump or implantable reservoir/infusion pump (without laminectomy); a comprehensive code, includes the following component codes:

- 62270 spinal puncture, lumbar, diagnostic

- 62272 spinal puncture, therapeutic, for drainage of spinal fluid

- 62273 injection, epidural, of blood or clot patch

- 62280 injection/infusion of neurolytic substance (subarachnoid)

- 62281 injection/infusion of neurolytic substance (epidural, cervical or thoracic)

- 62282 injection/infusion of neurolytic substance; epidural lumbar, sacral (caudal).

- 62310 injection, single, epidural or subarachnoid; cervical or thoracic

- 62311 injection, single; epidural, lumbar sacral (caudal)

- 62318 catheter placement, continuous infusion or intermittent bolus; epidural or subarachnoid; cervical or thoracic

- 62319 catheter placement, continuous infusion or intermittent bolus; epidural, lumbar, sacral (caudal)

- 64479 transforaminal epidural; cervical or thoracic, single level

- 64483 transforaminal epidural; lumbar or sacral, single level

- 69990 use of operating microscope

Mutually exclusive codes: None

- CPT 62351 - implantation, revision or repositioning of tunneled intrathecal or epidural catheter, for longterm medication administration via an external pump or implantable reservoir/infusion pump (with laminectomy); a comprehensive code, includes the following component codes:

- 62280 injection/infusion of neurolytic substance (subarachnoid)

- 62281 injection/infusion of neurolytic substance (epidural, cervical or thoracic)

- 62282 injection/infusion of neurolytic substance; 
epidural lumbar, sacral (caudal).

- 62310 injection, single, epidural or subarachnoid; cervical or thoracic

- 62311 injection, single; epidural, lumbar sacral (caudal)

- 62318 catheter placement, continuous infusion or intermittent bolus; epidural or subarachnoid; cervical or thoracic

- 62319 catheter placement, continuous infusion or intermittent bolus; epidural, lumbar, sacral (caudal)

- 62350 implantation, revision or repositioning of tunneled intrathecal or epidural catheter, for longterm medication administration via an external pump or implantable reservoir/infusion pump (without laminectomy)

- 64479 transforaminal epidural; cervical or thoracic, single level

- 64483 transforaminal epidural; lumbar or sacral, single level

- 69990 use of operating microscope

Mutually exclusive codes: None

- CPT 62355 - removal of previously implanted intrathecal or epidural catheter; a comprehensive code, includes the following component codes:

- 62270 spinal puncture, lumbar, diagnostic

- 62272 spinal puncture, therapeutic, for drainage of spinal fluid

- 62310 injection, single, epidural or subarachnoid; cervical or thoracic

- 62311 injection, single; epidural, lumbar sacral (caudal)

- 62318 catheter placement, continuous infusion or intermittent bolus; epidural or subarachnoid; cervical or thoracic

- 62319 catheter placement, continuous infusion or intermittent bolus; epidural, lumbar, sacral (caudal)

- 64479 transforaminal epidural; cervical or thoracic, single level

- 64483 transforaminal epidural; lumbar or sacral, single level

- 69990 use of operating microscope

Mutually exclusive codes:

- 62350 implantation, revision or repositioning of tunneled intrathecal or epidural catheter, for longterm medication administration via an external pump or implantable reservoir/infusion pump (without laminectomy)
- 62351 implantation, revision or repositioning of tunneled intrathecal or epidural catheter, for longterm medication administration via an external pump or implantable reservoir/infusion pump (with laminectomy)

- CPT 62360 - implantation or replacement of device for intrathecal or epidural drug infusion (subcutaneous reservoir); a comprehensive code, includes the following component codes:

- 62270 spinal puncture, lumbar, diagnostic

- 62272 spinal puncture, therapeutic, for drainage of spinal fluid

- 62273 injection, epidural, of blood or clot patch

- 62310 injection, single, epidural or subarachnoid; cervical or thoracic

- 62311 injection, single; epidural, lumbar sacral (caudal)

- 62318 catheter placement, continuous infusion or intermittent bolus; epidural or subarachnoid; cervical or thoracic

- 62319 catheter placement, continuous infusion or intermittent bolus; epidural, lumbar, sacral (caudal)

- 62367 electronic analysis of programmable, implanted pump for intrathecal or epidural drug infusion (without programming)

- 62368 electronic analysis of programmable, implanted pump for intrathecal or epidural drug infusion (with reprogramming)

- 64479 transforaminal epidural; cervical or thoracic, single level

- 64483 transforaminal epidural; lumbar or sacral, single level

- 69990 use of operating microscope

Mutually exclusive codes:

- 62280 injection/infusion of neurolytic substance (subarachnoid)

- 62281 injection/infusion of neurolytic substance (epidural, cervical or thoracic)

- 62282 injection/infusion of neurolytic substance; epidural lumbar, sacral (caudal).

- 62362 implantation of replacement of device for intrathecal or epidural drug infusion; programmable pump, including preparation of pump, with or without programming

- CPT 62361 - implantation or replacement of device for intrathecal or epidural drug infusion (nonprogrammable pump); a comprehensive code, 
includes the following component codes:

- 62270 spinal puncture, lumbar, diagnostic

- 62272 spinal puncture, therapeutic, for drainage of spinal fluid

- 62273 injection, epidural, of blood or clot patch

- 62310 injection, single; epidural or subarachnoid; cervical or thoracic

- 62311 injection, single; epidural, lumbar, sacral (caudal)

- 62318 catheter placement, continuous infusion or intermittent bolus; epidural or subarachnoid; cervical or thoracic

- 62319 catheter placement, continuous infusion or intermittent bolus; epidural, lumbar, sacral (caudal)

- 62367 electronic analysis of programmable, implanted pump for intrathecal or epidural drug infusion (without programming)

- 62368 electronic analysis of programmable, implanted pump for intrathecal or epidural drug infusion (with reprogramming)

- 64479 transforaminal epidural; cervical or thoracic, single level

- 64483 transforaminal epidural; lumbar or sacral, single level

- 69990 use of operating microscope

Mutually exclusive codes:

- 62280 injection/infusion of neurolytic substance (subarachnoid)

- 62281 injection/infusion of neurolytic substance (epidural, cervical or thoracic)

- 62282 injection/infusion of neurolytic substance; epidural lumbar, sacral (caudal).

- 62360 implantation or replacement of device for intrathecal or epidural drug infusion; subcutaneous reservoir

- CPT 62362 implantation or replacement of device for intrathecal or epidural drug infusion (programmable pump); a comprehensive code, a comprehensive code, includes the following component codes:

- 62270 spinal puncture, lumbar, diagnostic

- 62272 spinal puncture, therapeutic

- 62273 injection, epidural, of blood or clot patch

- 62310 cervical epidural

- 62311 lumbar epidural

- 62318 catheter placement, continuous infusion or intermittent bolus; epidural or subarachnoid; cervical or thoracic

- 62319 catheter placement, continuous infusion or intermittent bolus; epidural, lumbar, sacral (caudal)

- 62361 implantation or replacement of device for intrathecal or epidural drug infusion (non-programmable pump)

- 62367 electronic analysis of programmable, implanted pump for intrathecal or epidural drug infusion (without programming)

- 62368 electronic analysis of programmable, implanted pump for intrathecal or epidural drug infusion (with reprogramming)

- 64479 injection, cervical transforaminal epidural

- 64483 injection, anesthetic agent and/or steroid, transforaminal epidural (lumbar or sacral, single level)

- 69990 use of operating microscope

Mutually exclusive codes:

- 62280 injection/infusion of neurolytic substance (subarachnoid)

- 62281 injection/infusion of neurolytic substance (epidural, cervical or thoracic)

- 62282 injection/infusion of neurolytic substance; epidural lumbar, sacral (caudal)

- CPT 62365 removal of subcutaneous reservoir or pump, previously implanted for intrathecal or epidural infusion; a comprehensive code, a comprehensive code, includes the following component codes:

- 62310 cervical epidural

- 62311 lumbar epidural

- 62318 catheter placement, continuous infusion or intermittent bolus; epidural or subarachnoid; cervical or thoracic

- 62319 catheter placement, continuous infusion or intermittent bolus; epidural, lumbar, sacral (caudal)

- 62367 electronic analysis of programmable, implanted pump for intrathecal or epidural drug infusion (without programming)

- 62368 electronic analysis of programmable, implanted pump for intrathecal or epidural drug infusion (with reprogramming)

- 64479 injection, cervical transforaminal epidural

- 64483 injection, anesthetic agent and/or steroid, transforaminal epidural (lumbar or sacral, single 
level)

- 69990 use of operating microscope

- 96520 refilling and maintenance of portable pump

Mutually exclusive codes:

- 62360 implantation or replacement of device for intrathecal or epidural drug infusion; subcutaneous reservoir

- 62361 implantation or replacement of device for intrathecal or epidural drug infusion (non-programmable pump)

- 62362 implantation or replacement of device for intrathecal or epidural drug infusion; (programmable pump, including preparation of pump, with or without programming)

- CPT 64400 - injection, anesthetic agent (trigeminal nerve, any division or branch); a comprehensive code, includes the following component codes:

- 20550 injection, tendon sheath, ligament, trigger points or ganglion cyst

- 69990 use of operating microscope

Mutually exclusive codes: None

- CPT 64405 - injection, anesthetic agent (greater occipital nerve); a comprehensive code, includes the following component codes:

- 20550 injection, tendon sheath, ligament, trigger points or ganglion cyst

- 69990 use of operating microscope

Mutually exclusive codes: None

- CPT 64413 - injection, anesthetic agent (trigeminal nerve, any division or branch); a comprehensive code, includes the following component codes:

- 20550 injection, tendon sheath, ligament, trigger points or ganglion cyst

- 69990 use of operating microscope

Mutually exclusive codes: None

- CPT 64415 - injection, anesthetic agent (brachial plexus); a comprehensive code, includes the following component codes:

- 20550 injection, tendon sheath, ligament, trigger points or ganglion cyst

- 69990 use of operating microscope

Mutually exclusive codes: None
- CPT 64417 - injection, anesthetic agent (axillary nerve); a comprehensive code, includes the following component codes:

- 20550 injection, tendon sheath, ligament, trigger points or ganglion cyst

- 69990 use of operating microscope

Mutually exclusive codes: None

- CPT 64418 - injection, anesthetic agent (suprascapular nerve); a comprehensive code, includes the following component codes:

- 20550 injection, tendon sheath, ligament, trigger points or ganglion cyst

- 69990 use of operating microscope

Mutually exclusive codes: None

- CPT 64420 - injection, anesthetic agent (intercostal nerve, single); a comprehensive code, includes the following component codes:

- 20550 injection, tendon sheath, ligament, trigger points or ganglion cyst

- 69990 use of operating microscope

Mutually exclusive codes: None

- CPT 64421 - injection, anesthetic agent (intercostal nerves, multiple, regional block); a comprehensive code, includes the following component codes:

- 20550 injection, tendon sheath, ligament, trigger points or ganglion cyst

- 64420 injection, anesthetic agent (intercostal nerve, single)

- 69990 use of operating microscope

Mutually exclusive codes: None

- CPT 64425 - injection, anesthetic agent (ilioinguinal, iliohypogastric nerves); a comprehensive code, includes the following component codes:

- 20550 injection, tendon sheath, ligament, trigger points or ganglion cyst

- 69990 use of operating microscope

Mutually exclusive codes: None

- CPT 64430 - injection, anesthetic agent (pudendal nerve); a comprehensive code, includes the following component codes: 
- 20550 injection, tendon sheath, ligament, trigger points or ganglion cyst

- 69990 use of operating microscope

Mutually exclusive codes: None

- CPT 64445 - injection, anesthetic agent (sciatic nerve); a comprehensive code, includes the following component codes:

- 20550 injection, tendon sheath, ligament, trigger points or ganglion cyst

- 69990 use of operating microscope

Mutually exclusive codes: None

- CPT 64470 - cervical/thoracic facet joint nerve block; a comprehensive code, includes the following component codes:

- 20550 injection, tendon sheath, ligament, trigger points or ganglion cyst

- 20600 injection; small joint, bursa or ganglion

- 20605 injection, intermediate joint, bursa or ganglion

- 20610 injection, major joint or bursa

- 36140 introduction of needle or intracatheter, extremity artery

- 76000-76003 fluoroscopy codes

Mutually exclusive codes: None

- CPT 64475 - injection, lumbar facet joint nerve block; a comprehensive code, includes the following component codes:

- 20550 injection, tendon sheath, ligament, trigger points or ganglion cyst

- 20600 injection; small joint, bursa or ganglion

- 20605 injection, intermediate joint, bursa or ganglion

- 20610 injection, major joint or bursa

- 36140 introduction of needle or intracatheter, extremity artery

- 69990 use of operating microscope

- 76000-76003 fluoroscopy codes

Mutually exclusive codes: None

- CPT 64479 - injection, cervical transforaminal epidural; a comprehensive code, includes the following component codes:

- 20550 injection, tendon sheath, ligament, trigger points or ganglion cyst
- 20605 injection, intermediate joint, bursa or ganglion

- 20610 injection, major joint or bursa

- 36140 introduction of needle or intracatheter, extremity artery

- 69990 use of operating microscope

- 76000-76003 fluoroscopy codes

Mutually exclusive codes: None

- CPT 64480 - injection, anesthetic agent and/or steroid, transforaminal epidural (cervical or thoracic, each additional level); a comprehensive code, includes the following component codes:

- 69990 use of operating microscope

Mutually exclusive codes: None

- CPT 64483 - injection, anesthetic agent and/or steroid, transforaminal epidural (lumbar or sacral, single level); a comprehensive code, includes the following component codes:

- 20550 injection, tendon sheath, ligament, trigger points or ganglion cyst

- 20605 injection, intermediate joint, bursa or ganglion

- 20610 injection, major joint or bursa

- 36140 introduction of needle or intracatheter, extremity artery

- 76000-76003 fluoroscopy codes

Mutually exclusive codes: None

- CPT 64505 - injection, anesthetic agent (sphenopalatine ganglion); a comprehensive code, includes the following component codes:

- 69990 use of operating microscope

Mutually exclusive codes: None

- CPT 64510 - injection, anesthetic agent (stellate ganglion) cervical sympathetic; a comprehensive code, includes the following component codes:

- 69990 use of operating microscope

Mutually exclusive codes: None

- CPT 64520 - injection, anesthetic agent (lumbar or thoracic) paravertebral sympathetic; a comprehensive code, includes the following component codes: 
- 69990 use of operating microscope

Mutually exclusive codes: None

- CPT 64530 - injection, anesthetic agent (celiac plexus, with or without radiologic monitoring); a comprehensive code, includes the following component codes:

- 69990 use of operating microscope

Mutually exclusive codes: None

- CPT 64600 - destruction by neurolytic agent, trigeminal nerve (supraorbital, infraorbital, mental, or inferior alveolar branch); a comprehensive code, includes the following component codes:

- 64400 injection, anesthetic agent (trigeminal nerve, any division or branch)

- 69990 use of operating microscope

Mutually exclusive codes: None

- CPT 64605 - destruction by neurolytic agent, trigeminal nerve (second and third division branches at foramen ovale); a comprehensive code, includes the following component codes:

- 69990 use of operating microscope

Mutually exclusive codes: None

- CPT 64610 - destruction by neurolytic agent, trigeminal nerve (second and third division branches at foramen ovale under radiologic monitoring); a comprehensive code, includes the following component codes:

- 64605 destruction by neurolytic agent, trigeminal nerve; second and third division branches at foramen ovale

- 69990 use of operating microscope

Mutually exclusive codes: None

- CPT 64612 - chemodenervation of muscle(s) (muscle(s) innervated by facial nerve); a comprehensive code, includes the following component codes:

- 64402 injection, anesthetic agent (facial nerve)

Mutually exclusive codes: None

- CPT 64613 - chemodenervation of muscle(s) (cervical spine muscle(s)); a comprehensive code, includes the following component codes:
- 69990 use of operating microscope

Mutually exclusive codes: None

- CPT 64620 - destruction by neurolytic agent (intercostal nerve); a comprehensive code, includes the following component codes:

- 64420 injection, anesthetic agent (intercostal nerve, single)

- 64421 injection, anesthetic agent (intercostal nerves, multiple, regional block)

- 69990 use of operating microscope

Mutually exclusive codes: None

- CPT 64622 - destruction by neurolytic agent, paravertebral facet joint nerve (lumbar or sacral, single level); a comprehensive code, includes the following component codes:

- 64475 lumbar facet joint nerve block

- 69990 use of operating microscope

- 76000-76003 fluoroscopy codes

Mutually exclusive codes: None

- CPT 64626 - destruction by neurolytic agent, paravertebral facet joint nerve (cervical or thoracic, single level); a comprehensive code, includes the following component codes:

- 64470 cervical paravertebral facet joint nerve block

- 69990 use of operating microscope

- 76000-76003 fluoroscopy codes

Mutually exclusive codes: None

- CPT 64630 - destruction by neurolytic agent, pudendal nerve; a comprehensive code, includes the following component codes:

- 64430 injection, anesthetic agent (pudendal nerve)

- 69990 use of operating microscope

Mutually exclusive codes: None

- CPT 64640 - destruction by neurolytic agent, other peripheral nerve or branch; a comprehensive code, includes the following component codes:

- 64405 injection, anesthetic agent (greater occipital nerve)

- 64408 injection, anesthetic agent (vagus nerve)

- 64410 injection, anesthetic agent (phrenic nerve) 
- 64412 injection, anesthetic agent (spinal accessory nerve)

- 64413 injection, anesthetic agent (cervical plexus)

- 64415 injection, anesthetic agent (brachial plexus)

- 64417 injection, anesthetic agent (axillary nerve)

- 64418 injection, anesthetic agent (suprascapular nerve)

- 64425 injection, anesthetic agent (ilioinguinal, iliohypogastric nerve)

- 64435 injection, anesthetic agent (paracervical [uterine] nerve)

- 64445 injection, anesthetic agent (sciatic nerve)

- 64450 injection, anesthetic agent (other peripheral nerve or branch)

- 69990 use of operating microscope

Mutually exclusive codes: None

- CPT 64680 - destruction by neurolytic agent, celiac plexus, with or without radiologic monitoring; a comprehensive code, includes the following component codes:

- 64530 celiac plexus block

- 69990 use of operating microscope

Mutually exclusive codes: None

- CPT 72275 - epidurography, radiological supervision and interpretation; a comprehensive code, includes the following component codes:

- 76000 fluoroscopy (separate procedure), up to 1 hour of physician time, other than 71023 or 71034

- 76001 fluoroscopy, physician time more than 1 hour, assisting a nonradiologic physician

- 76003 fluoroscopic guidance for needle placement

- 76005 fluoroscopic guidance and localization of needle or catheter tip for spine or paraspinous diagnostic or therapeutic injection procedures

Mutually exclusive codes: None

- CPT 72285 - diskography, cervical or thoracic, radiological supervision and interpretation; a comprehensive code, includes the following component codes:

- 76005 fluoroscopic guidance and localization of needle or catheter tip for spine or paraspinous diagnostic or therapeutic injection procedures

Mutually exclusive codes: None
- CPT 72295 - diskography, lumbar, radiological supervision and interpretation; a comprehensive code, includes the following component codes:

- 76005 fluoroscopic guidance and localization of needle or catheter tip for spine or paraspinous diagnostic or therapeutic injection procedures

\section{Mutually exclusive codes: None}

\section{CONCLUSION}

The CMS (HCFA), to help ensure that providers across various jurisdictions receive like payments for the same services, use the same codes and provide similar documentation for services performed, created the NCCC, established the National Correct Coding Policy, and implemented the "CCI." Interventional pain physicians, like the rest of the medical community, are deeply entangled in National Correct Coding Policy and CCI. It is imperative for interventional pain physicians to avoid incorrect coding, either by unbundling or upcoding, and to understand National Correct Coding Policy and Correct Coding Edits, some of which are described here.

\section{REFERENCES}

1. Manchikanti L. The impact of National Correct Coding Policy on interventional pain management. Pain Physician 1999; 2:33-45.

2. National Correct Coding Manual 2001. Medical Management Institute, Alpharetta; 2001.

3. $\mathrm{CCI}$ edits require expertise in the face of voluminous outpatient, coding changes. HCPCS Report 2000; 14:1-5.

4. Current Procedural Terminology. CPT 1999, Chicago, American Medical Association, 1998.

5. Current Procedural Terminology. CPT 2000, Chicago, American Medical Association, 1999.

6. Manchikanti L. Evaluation and management services in interventional pain practice: Doing it right! Pain Physician 2000; 3:322-341

7. Manchikanti L. The role of evaluation and management services in pain management. Pain Physician 1999; 2:10-32.

8. Manchikanti L. Appropriate documentation, billing and coding in interventional pain practice. Pain Physician 2000; 3:218-236.

9. CCI version 6.1 Errata. Coding and Medicare Updates. 2000: May 1-2.

10. Manchikanti L. CPT 2000: Interventional pain management coding in the new millennium. Pain Physician 2000; 3:73-85. 